\title{
Tortoise and Turtle Extension Lifespan in Related to Branched-Chain and Sulphur Contain Amino Acids: A Review
}

\author{
Md Serajul Islam ${ }^{1,2}$, Hongxin Wang, ${ }^{1,2 *}$ Chaoyang $\mathrm{Ma}^{1,2}$, Juanhui Jing ${ }^{2}$, Duxiaojing ${ }^{2}$, Noyazzesh \\ Hossain ${ }^{3}$, Wang $\mathrm{Li}^{2}$ and $\mathrm{Fu}$ An wei $^{4}$ \\ ${ }^{1}$ State key Laboratory of Food Science and Technology, Jiangnan University, Wuxi, P.R. China
}

${ }^{2}$ National Engineering Research Center for Functional Food, Jiangnan University, Wuxi, P.R. China

${ }^{3}$ University of Eastern Finland, Joensuu, Kuopio, Finland

${ }^{4}$ Guangxi Yitaining Biotechnology Co. Ltd., P.R. China

*Corresponding author: Hongxin Wang, State key Laboratory of Food Science and Technology, National Engineering Research

Center for Functional Food, Jiangnan University, Wuxi-214122, P.R. China

ARTICLE INFO

Received: 㸷 June 08, 2020

Published: 慧 June 17, 2020

Citation: Md. Serajul Islam, Hongxin Wang, Chaoyang Ma, Juanhui Jing, Duxiaojing, Noyazzesh Hossain, et al. Tortoise and Turtle Extension Lifespan in Related to Branched-Chain and Sulphur Contain Amino Acids: A Review. Biomed J Sci \& Tech Res 28(2)-2020. BJSTR. MS.ID.004634.

\author{
ABSTRACT
}

Branched-chain amino acids (BCAAs) are the direct effect on the initiation of mRNA translation and potent at activating Target of Rapamycin (TOR), which probable have relation with aging factors. Dietary restrictions, prolonged starvation and mutations that cause feeding defects are factors that stimulate longevity. Low/medium temperature condition is also important factors that influence ageing. Protein involves to metabolism system so that may be leucine and methionine amino acid relation to long life aging factors. Turtles and Tortoises haves low metabolism, very slow-moving, relaxed creatures that play role to aging factors in animals and also shell to protect from predation and physical other threats. Therefore, intensive research efforts are highly needed to long life Tortoise/Turtle related to protein metabolism and isolation of age related specific amino acids sequence.

Keywords: Tortoises/Turtles, BCAAs; Sulfur contains amino acid; Long Life Aging Factors; Protein Metabolism

\section{Introduction}

There are around 350 Turtle/Tortoise living species worldwide in 13 Families and $>90$ Genera. Of these, 49 are land, 8 species are marine, and $\leq 250$ are fresh water to semi aquatic [1].Turtle and Tortoise belongs to completely different Family but Orders are same. Turtles mostly dwell in water and Tortoises generally dwell on land. Beside there is a huge difference in their average lifespan. Tortoise/Turtles contain high amount of protein and with micronutrient (Calcium, Phosphorus, Vitamin-B12, Vitamin-K, and Vitamin-D), that makes body building and project some disease. Kienzle, Kopsch [2] finding protein content of the body without shell was $74.6 \%$ in Tortoises (Testudohermanni,Testudohorsfieldii) and aquatic Turtles (Rhinoclemmispulcherrima; juvenile) was $62.7 \%$ according to dry basis. Li, Tang [3] who reported some species, such as Cuora trifasciata, are to have additional properties in curing cancer and other hard-to-heal diseases. Methionine is a powerful antioxidant, and sulfur contains amino acids helps to neutralize free radicals that are formed of various metabolic processes in the body. Balch [4] reported, if not neutralized free radicals interact with DNA and the proteins in healthy cells then damage tissues and organs (skin, nails, heart, liver, and lungs). Methionine also plays an important role in the proper functioning of the immune system.

Van De Poll, Dejong [5] informed that high methionine levels in the body increase the levels of other amino acids such as glutathione, homo-cysteine, and Taurine, while glutathione and taurine that improve immune function and suppress inflammatory reactions. Amino acids are an absolutely critical for proper 
immunological, neurological, and gastrointestinal functions, which are used to construct enzymes, antibodies, immunoglobulins, neurotransmitters, hormones and also an important part of the detoxification process. Methionine, taurine, cysteine, and homocysteine are the sulfur-containing amino acids, while methionine and cysteine both are incorporated into proteins buthomo-cysteine and taurine play vital physiological roles. The body can make cysteine from methionine, for this reason methionine is a dietary essential [6]. The multifunctional role of methionine includes its participation in the synthesis of carnitine, creatine, glutathione, polyamines, nucleic acids, catechol-amines (neurotransmitters), as well as serving as a substrate for protein synthesis.

\section{Some Research Findings and Related Hypothesis- Why Long Life of Tortoises/Turtles?}

One of the most accepted hypotheses concerning the longevity of Turtles/Tortoises due to their slow metabolism. Metabolism generally refers to the physical and chemical processes that go on inside a living being in order to keep it alive. Panda [7] informed Tortoise/Turtle is a tranquil nature and diligence, or perhaps its hard shell/ calipash that protects from most physical dangers. It assumes that, having a slow metabolism means that burn energy at a very slow rate, which further opens up other opportunities for longevity. First of all, they can survive for a really long time without food, patient process through which they break down the ingested food and convert it into energy. In addition, Turtles/Tortoises are very slow-moving and relaxed creatures. The longevity of Tortoises can be complete without mentioning the enviable armor that covers almost their entire body [8]. Turtles also realize the importance of a strict vegetarian diet; they usually eat only greens like lettuce, soaked gram (chickpea), carrots, wheat bran, bread, grass and salt at mealtime that staying clear of fat and cholesterol. The oldest known Tortoise on record, Adwaita is one of the longest-living animals in modern times, Adwaita lived to be 255years old. Also, the Aldabra Giant Tortoise had been given to the India Alipore Zoo in 1876 that was assumed to be 150 years old at his death in 2006 [9].

Some land Tortoises/Turtles for instance the Galapagos tortoise can survive for 4-5 months without access food or water. Beside, desert Tortoise of North America can survive for a year with no hydration. This reason may be Tortoise's water intake comes from the flowers and greens dietthat are stored in his/her body. Even water in his/her bladder can be called upon to hydrate this land Turtle in moments of crisis.

\section{Literature Review}

The ability of methionine (Met) restriction to minimize respiratory electron leak contribute prominently to its impact on longevity, In addition, glutamate supplementation extended chronological lifespan [10]. Consistent with the ability of glutamate to extend lifespan, deletion of genes involved in converting glutamate to gamma-aminobutyric acid (GABA) increased replicative lifespan [11] and led to increased conversion of glutamate to $\alpha$-ketoglutarate and other TCA cycle intermediates, which may be involved in lifespan extension by maintaining mitochondrial respiratory function. Increased levels of amino acids, especially leucine and activate the TOR kinase, which leads to an increased rate of translation $[12,13]$. The cumulative impact of mitochondrial oxidant stress on the structure and function of mitochondria that can play a key role in the aging process, and progressive mutation or deletion of mitochondrial DNA contributes importantly to aging [14]. Several screens in model organisms have concerned nuclearencoded mitochondrial proteins in the regulation of lifespan with how food availability and metabolism are molecularly coupled to lifespan [15]. Beside, Inhibition of the TOR kinase with Rapamycinor expressing a dominant negative p70-S6 kinase, where a kinase downstream of TOR as a result extended organismal longevity [16, 17]. Metabolism of sulfur containing amino acids are the essential for DR-mediated longevity in Drosophila [18], but supplementation of methionine or cysteine failed to extend lifespan in fully fed Drosophila $[19,20]$.

However, supplementing casein and methionine together led to lifespan extension [19]. High-protein diets maximize reproduction and shorten lifespan, and circulating branched-chain amino acids (BCAAs) (leucine, isoleucine, and valine) in particular have been implicated in promoting aging [21, 22]. BCAAs are especially potent at activating Target of Rapamycin (TOR) [23], a central cellular pathway of protein sensing and growth. Leucine is the most prevalent of the three BCAAs, which shown to signal the TOR pathway via sestrin2 [24]. In addition, leucine is oxidized to make ATP and carbon- 1 is the first carbon released in $\mathrm{CO}_{2}$ (when NADH is produced). The $\mathrm{CO}_{2}$ is exhaled and is detected by our oxidation assay. Because of its tight association with TOR and the clear interpretation of its oxidation, we chose to investigate oxidation and allocation of dietary 13C-1-leucine. The Respiratory Exchange Ratios (RERs) provides an unambiguous indication of fuel substrate when only carbohydrates are metabolized (RER=1.0) or when only lipids are metabolized (RER=0.7). All intermediate values are either a combination of the two or indicate protein metabolism (RER=0.8). Therefore, it measured the oxidation of amino acid (leucine), lipid (oleic acid), and carbohydrate (glucose). Ovariectomy and dietary restriction each increased oxidation of dietary leucine. Dietary leucine mayplay a special role in aging because amino acids stimulate cellular growth and temperature is also a growth condition that influences ageing.

Speeding oxidation of leucine may attenuate cellular growth [25]. However, it has been shown that methionine or tryptophan restriction extends lifespan in higher eukaryotes and increased proline or tryptophan levels increase longevity in Caenorhabditis elegans. In addition, leucine strongly activates the TOR signaling pathway, which when inhibited increases lifespan. The overexpression of DAF-16 in wild-type animals only slightly 
increases their lifespan, indicating that solely increasing levels of DAF-16 is not sufficient to significantly extend Longevity [26].

\section{Conclusion}

In conclusions, a number of observations in a wide range of experimental models have linked metabolism to aging. BCAAs and methionine mostly involve to long life aging factors because leucine is an active complex amino acid and methionine increase growth hormone which increase extension aging. Tortoises and Turtles having a slow metabolism that means they burn energy at a very slow rate as a result further opens up other opportunities for longevity.

\section{Conflict of Interest}

The authors declare no conflicts of interest.

\section{Acknowledgement}

This work was supported by national first-class discipline program of Food Science and Technology (JUFSTR20180204). We also extend our sincere gratitude to the staff and students of the research center of Food Nutrition and Functional Factors.

\section{References}

1. Mitchell JC, K Buhlmann (2005) Sustaining America's Aquatic Biodiversity. Turtle Biodiversity and Conservation 425-529.

2. Kienzle E (2006) Chemical composition of turtles and tortoises. The Journal of nutrition 136(7): p. 2053S-2054S.

3. Li G, D Tang, K Fang (2009) An analysis of amino acids in the muscles of Cuora trifasciata. Sichuan journal of zoology 19(3): p. 165-166.

4. Balch PA (2006) Prescription for nutritional healing. Penguin.

5. Van De Poll MC, CH Dejong, PB (2006) Soeters, Adequate range for sulfur-containing amino acids and biomarkers for their excess: lessons from enteral and parenteral nutrition. The Journal of nutrition 136(6): p. $1694 \mathrm{~S}-1700 \mathrm{~S}$.

6. Di Pasquale MG (2007) Amino acids and proteins for the athlete: The anabolic edge. CRC Press.

7. Panda KF (2008) “There are no accidents.” Master Oogway, senior master of the Jade Palace. Kung Fu Panda (2008 Dreamworks Animation).

8. Carr A (2018) Handbook of turtles: The turtles of the United States, Canada, and Baja California. 2018: Cornell University Press.

9. Allan GB (2011) White people have souls.

10. Wu Z (2013) Independent and additive effects of glutamic acid and methionine on yeast longevity. PloS one 8(11): e79319.

11. Kamei Y, Takayuki Tamura, Ryo Yoshida, Shinji Ohta, Eiichiro Fukusaki, et al. (2011) GABA metabolism pathway genes, UGA1 and GAD1, regulate replicative lifespan in Saccharomyces cerevisiae. Biochemical and biophysical research communications 407(1): p. 185-190.
12. Ban H, Kaori Shigemitsu, Tomoki Yamatsuji, Minoru Haisa, Tohru Nakajo, et al. (2004) Arginine and Leucine regulate p70 S6 kinase and 4E-BP1 in intestinal epithelial cells. International journal of molecular medicine, 2004. 13(4): p. 537-543.

13. Hara K, K Yonezawa, QP Weng, MT Kozlowski, C Belham, et al. (1998) Amino acid sufficiency and $m$ TOR regulate $p 70$ S6 kinase and eIF-4E BP1 through a common effector mechanism. Journal of Biological Chemistry 273(23): p. 14484-14494.

14. Gredilla R, G Barja (2005) Minireview: The role of oxidative stress in relation to caloric restriction and longevity. Endocrinology 146(9): p. 3713-3717.

15. Finkel $\mathrm{T}$ (2015) The metabolic regulation of aging. Nature medicine 21(12): p. 1416.

16. Kapahi P, Brian M Zid, Tony Harper, Daniel Koslover, Viveca Sapin, et al. (2004) Regulation of lifespan in Drosophila by modulation of genes in the TOR signaling pathway. Current Biology 14(10): p. 885-890.

17. Moskalev A, M Shaposhnikov (2010) Pharmacological inhibition of phosphoinositide 3 and TOR kinases improves survival of Drosophila melanogaster. Rejuvenation research 13(2-3): p. 246-247.

18. Kabil H, Omer Kabil, Ruma Banerjee, Lawrence G Harshman, Scott D Pletcher, et al. (2011) Increased transsulfuration mediates longevity and dietary restriction in Drosophila. Proceedings of the National Academy of Sciences 108(40): p. 16831-16836.

19. Massie HR, T Williams (1985) Effect of sulfur-containing compounds on the life span of Drosophila. Age 8(4): p. 128-135.

20. Zajitschek F, (2013) Interactive effects of sex, social environment, dietary restriction, and methionine on survival and reproduction in fruit flies. Age 35(4): p. 1193-1204.

21. Mold JE, Jakob Michaëlsson, Trevor D Burt, Marcus O Muench, Karen P Beckerman, et al. (2008) Maternal alloantigens promote the development of tolerogenic fetal regulatory $\mathrm{T}$ cells in utero. Science 322(5907): p. 1562-1565.

22. Solon Biet SM, Aisling C McMahon, J William O Ballard, Kari Ruohonen, Lindsay $\mathrm{E} \mathrm{Wu}$, et al. (2014) The ratio of macronutrients, not caloric intake, dictates cardiometabolic health, aging, and longevity in ad libitum-fed mice. Cell metabolism 19(3): p. 418-430.

23. Chotechuang $N$ (2010) The role of amino acids in liver protein metabolism under a high protein diet: Identification of amino acids signal and associated transduction pathways.

24. Wolfson RL, Lynne Chantranupong, Robert A Saxton, Kuang Shen, Sonia M Scaria, et al. (2016) Sestrin2 is a leucine sensor for the mTORC1 pathway. Science 351(6268): p. 43-48.

25. Heck MJ, Mirna Pehlivanovic, Jennifer U Purcell, Daniel A Hahn, John D Hatle, et al. (2017) Life-extending dietary restriction reduces oxidative damage of proteins in grasshoppers but does not alter allocation of ingested nitrogen to somatic tissues. The Journals of Gerontology: Series A 72(5): p. 616-623.

26. Henderson ST, TE Johnson (2001) daf-16 integrates developmental and environmental inputs to mediate aging in the nematode Caenorhabditis elegans. Current Biology 11(24): p. 1975-1980. 
ISSN: 2574-1241

DOI: 10.26717/BJSTR.2020.28.004634

Hongxin Wang. Biomed J Sci \& Tech Res

(C) (i) This work is licensed under Creative

Submission Link: https://biomedres.us/submit-manuscript.php

$\begin{array}{ll}\text { BIOMEDICAL } & \text { Assets of Publishing with us } \\ \text { RESEARCHES } & \text { - Global archiving of articles } \\ \text { - Immediate, unrestricted online access } & \text { - Rigorous Peer Review Process } \\ & \text { - Authors Retain Copyrights } \\ & \end{array}$

